\title{
Bacteroides salyersiae
}

National Cancer Institute

\section{Source}

National Cancer Institute. Bacteroides salyersiae. NCI Thesaurus. Code C122182.

A species of anaerobic, Gram-negative, non-motile bacterium in the family

Bacteroidaceae. B. salyersiae is a normal part of the gut flora and an opportunistic pathogen 